\title{
Dirac-harmonic maps from degenerating spin surfaces I: the Neveu-Schwarz case
}

\author{
Miaomiao Zhu
}

Received: 18 March 2008 / Accepted: 23 July 2008 / Published online: 15 August 2008

(C) The Author(s) 2008. This article is published with open access at Springerlink.com

\begin{abstract}
We study Dirac-harmonic maps from degenerating spin surfaces with uniformly bounded energy and show the so-called generalized energy identity in the case that the domain converges to a spin surface with only Neveu-Schwarz type nodes. We find condition that is both necessary and sufficient for the $W^{1,2} \times L^{4}$ modulo bubbles compactness of a sequence of such maps.
\end{abstract}

Mathematics Subject Classification (2000) $\quad 58 \mathrm{~J} 05 \cdot 53 \mathrm{C} 27$

\section{Introduction}

The notion of Dirac-harmonic maps was first introduced in [6]. Motivated by the supersymmetric nonlinear sigma model from quantum field theory [8], Dirac-harmonic maps are defined as solutions of a system of harmonic-type equations coupled with Dirac-type equations. As is done in the theory of minimal surfaces in Riemannian manifolds and pseudoholomorphic curves in symplectic geometry, construction of geometric invariants from the solution spaces is expected. This supersymmetric model is introduced in such a natural way that most fundamental features of two-dimensional harmonic maps are preserved. Following the approach of Sacks and Uhlenbeck [17], Chen et al. [5,6] developed the "blow-up" analysis for Dirac-harmonic maps and established the energy identity for a sequence of Diracharmonic maps with uniformly bounded energy ([5] for spherical targets and [20] for general targets), which gives the $W^{1,2} \times L^{4}$ modulo bubbles compactness of the solution space for a fixed spin surface. A natural question then is whether such compactness is preserved if we allow the domain surface to vary.

Supported by IMPRS "Mathematics in the Sciences" and the Klaus Tschira Foundation.

M. Zhu $(\bowtie)$

Max Planck Institute for Mathematics in the Sciences, Inselstr. 22-26, 04103 Leipzig, Germany

e-mail: Miaomiao.Zhu@mis.mpg.de 
To state the problem more precisely, we consider a sequence of smooth Dirac-harmonic maps

$$
\left(\phi_{n}, \psi_{n}\right):\left(M_{n}, h_{n}, c_{n}, \mathfrak{S}_{n}\right) \rightarrow(N, g),
$$

with uniformly bounded energy $E\left(\phi_{n}, \psi_{n}, M_{n}\right) \leq \Lambda<\infty$. Here $(N, g)$ is a compact Riemannian manifold with metric $g$ and $\left(M_{n}, h_{n}, c_{n}, \mathfrak{S}_{n}\right)$ is a sequence of closed hyperbolic Riemann surfaces of genus $g>1$ with hyperbolic metrics $h_{n}$, compatible complex structures $c_{n}$ and spin structures $\mathfrak{S}_{n}$.

In this paper, we first prove the energy identity for the sequence (1.1) when the domain surface varies in a compact region. Then, we show the so-called generalized energy identity for the sequence when the domain surface degenerates to a spin surface with only NeveuSchwarz type nodes. The necessary and sufficient condition for the $W^{1,2} \times L^{4}$ modulo bubbles compactness of such sequences is a direct consequence of the generalized energy identity.

Let us consider the simpler case that $\left(M_{n}, h_{n}, c_{n}\right)$ converges to a compact hyperbolic Riemann surface $(M, h, c)$ of the same topological type. Then there exists a sequence of diffeomorphisms $\tau_{n}: M \rightarrow M_{n}$ such that $\left(\tau_{n}^{*} h_{n}, \tau_{n}^{*} c_{n}\right)$ converges to $(h, c)$ in $C^{\infty}$. After passing to a subsequence, we can assume that the pull-back of $\mathfrak{S}_{n}$ via $\tau_{n}$ is a fixed spin structure on $M$. Let us denote it by $\mathfrak{S}$. Then, we can fix the spinor bundle $\Sigma M$ and think of the hyperbolic metrics $h_{n}$ and the compatible complex structures $c_{n}$ as all living on the limit surface $M$ and converging in $C^{\infty}$ to $h$ and $c$, respectively. Let $\nabla_{n}$ be the connection on $\Sigma M$ coming from $h_{n}$ and $\nabla$ the connection on $\Sigma M$ coming from $h$. Replaced by the pullbacks, we can think of $\left(\phi_{n}, \psi_{n}\right)$ as a sequence of Dirac-harmonic maps defined on $\left(M, h_{n}, c_{n}, \mathfrak{S}\right)$ with respect to $\left(c_{n}, \nabla_{n}\right)$. Then we prove the following energy identity for Dirac-harmonic maps from non-degenerating spin surfaces:

Theorem 1.1 Assumptions and notations as above. Then there exist finitely many blowup points $\left\{x_{1}, x_{2}, \ldots, x_{I}\right\}$, finitely many Dirac-harmonic maps $\left(\sigma^{i, l}, \xi^{i, l}\right): S^{2} \rightarrow N, i=1$, $2, \ldots, I ; l=1,2, \ldots, L_{i}$, and a Dirac-harmonic map $(\phi, \psi):(M, h, \mathfrak{S}) \rightarrow N$ such that after selection of a subsequence, $\left(\phi_{n}, \psi_{n}\right)$ converges to $(\phi, \psi)$ in $C_{l o c}^{\infty} \times C_{l o c}^{\infty}$ on $M \backslash\left\{x_{1}, x_{2}, \ldots, x_{I}\right\}$ and the following hold

$$
\begin{aligned}
& \lim _{n \rightarrow \infty} E\left(\phi_{n}\right)=E(\phi)+\sum_{i=1}^{I} \sum_{l=1}^{L_{i}} E\left(\sigma^{i, l}\right), \\
& \lim _{n \rightarrow \infty} E\left(\psi_{n}\right)=E(\psi)+\sum_{i=1}^{I} \sum_{l=1}^{L_{i}} E\left(\xi^{i, l}\right) .
\end{aligned}
$$

To continue the discussions, we recall that the Hopf quadratic differential associated to a two-dimensional harmonic map plays an important role in establishing the so-called generalized energy identity for harmonic maps from degenerating Riemann surfaces [22]. It is observed in [6] that there is a generalization of the notion of Hopf differential for a two dimensional Dirac-harmonic map. Let $(\phi, \psi)$ be a Dirac-harmonic map defined on a standard cylinder $P=\left[t_{1}, t_{2}\right] \times S^{1}$ with flat metric $d s^{2}=d t^{2}+d \theta^{2}$ and $T(\phi, \psi)(d t+i d \theta)^{2}$ the generalized Hopf differential of $(\phi, \psi)$ on $P$. Then the following integral

$$
\int_{\{t\} \times S^{1}} T(\phi, \psi) d \theta
$$

is a complex number which is independent of $t \in\left[T_{1}, T_{2}\right]$. Let us denote it by $\alpha=\alpha(\phi, \psi, P)$. 
Now we consider the case that $\left(M_{n}, h_{n}, c_{n}\right)$ degenerates to a hyperbolic Riemann surface $(M, h, c)$ by collapsing $p(1 \leq p \leq 3 g-3)$ pairwise disjoint simple closed geodesics $\gamma_{n}^{j}$ of length $l_{n}^{j}, j=1,2, \ldots, p$. For each $j$, the geodesics $\gamma_{n}^{j}$ degenerate into a pair of punctures $\left(\mathcal{E}^{j, 1}, \mathcal{E}^{j, 2}\right)$. Let $P_{n}^{j}$ be the standard cylindrical collar about $\gamma_{n}^{j}$. Then, we associate the sequence $\left(\phi_{n}, \psi_{n}, M_{n}\right)$ with a sequence of $p$-tuples $\left(\alpha_{n}^{1}, \ldots, \alpha_{n}^{p}\right)$, where $\alpha_{n}^{j}:=\alpha\left(\phi_{n}, \psi_{n}, P_{n}^{j}\right) \in \mathbb{C}$ are the quantities defined via (1.4).

By taking subsequences, we can assume that the pull back of $\mathfrak{S}_{n}$ via $\tau_{n}$ is a fixed spin structure $\mathfrak{S}$ on $M$. Note that $M$ has $p$ pairs of punctures. We require the following additional assumption:

All punctures of the limit spin surface $(M, \mathfrak{S})$ are of Neveu-Schwarz type.

Then $\mathfrak{S}$ extends to some spin structure $\overline{\mathfrak{S}}$ on $\bar{M}$, where $\bar{M}$ is the surface obtained by adding a point at each puncture of $M$. As before, we think of the hyperbolic metrics and the compatible complex structures $\left(h_{n}, c_{n}\right)$ as all living on the limit surface $M$ and converging in $C_{l o c}^{\infty}$ to $(h, c)$. Thus, $\left(\phi_{n}, \psi_{n}\right)$ becomes a sequence of Dirac-harmonic maps defined on $\left(M, h_{n}, c_{n}, \mathfrak{S}\right)$. Then we show the following generalized energy identity for Dirac-harmonic maps from degenerating spin surfaces:

Theorem 1.2 Assumptions and notations as above. Then there exist finitely many blow-up points $\left\{x_{1}, x_{2}, \ldots, x_{I}\right\}$ which are away from the punctures $\left\{\left(\mathcal{E}^{j, 1}, \mathcal{E}^{j, 2}\right), j=1,2, \ldots, p\right\}$ and finitely many Dirac-harmonic maps

$(\phi, \psi):(\bar{M}, \bar{c}, \overline{\mathfrak{S}}) \rightarrow N$, where $(\bar{M}, \bar{c}, \overline{\mathfrak{S}})$ is the normalization of $(M, c, \mathfrak{S})$,

$\left(\sigma^{i, l}, \xi^{i, l}\right): S^{2} \rightarrow N, l=1,2, \ldots, L_{i}$, near the ith blow-up point $x_{i}$,

$\left(\omega^{j, k}, \zeta^{j, k}\right): S^{2} \rightarrow N, k=1,2, \ldots, K_{j}$, near the $j$ th pair of punctures $\left(\mathcal{E}^{j, 1}, \mathcal{E}^{j, 2}\right)$,

such that after selection of a subsequence, $\left(\phi_{n}, \psi_{n}\right)$ converges to $(\phi, \psi)$ in $C_{l o c}^{\infty} \times C_{l o c}^{\infty}$ on $M \backslash\left\{x_{1}, x_{2}, \ldots, x_{I}\right\}$, and the following holds:

$$
\begin{aligned}
& \lim _{n \rightarrow \infty} E\left(\phi_{n}\right)=E(\phi)+\sum_{i=1}^{I} \sum_{l=1}^{L_{i}} E\left(\sigma^{i, l}\right)+\sum_{j=1}^{p} \sum_{k=1}^{K_{j}} E\left(\omega^{j, k}\right)+\sum_{j=1}^{p} \lim _{n \rightarrow \infty}\left|\operatorname{Re} \alpha_{n}^{j}\right| \cdot \frac{2 \pi^{2}}{l_{n}^{j}}, \\
& \lim _{n \rightarrow \infty} E\left(\psi_{n}\right)=E(\psi)+\sum_{i=1}^{I} \sum_{l=1}^{L_{i}} E\left(\xi^{i, l}\right)+\sum_{j=1}^{p} \sum_{k=1}^{K_{j}} E\left(\zeta^{j, k}\right) .
\end{aligned}
$$

As a corollary, we have

Corollary 1.1 Assumptions and notations as in Theorem 1.2. Then $\left(\Phi_{n}, \Psi_{n}, M_{n}\right)$ subconverge in $W^{1,2} \times L^{4}$ modulo bubbles, i.e., in the limit, the necks contain no energy if and only if

$$
\liminf _{n \rightarrow \infty}\left|\operatorname{Re} \alpha_{n}^{j}\right| \cdot \frac{2 \pi^{2}}{l_{n}^{j}}=0, \quad j=1,2, \ldots, p .
$$

For the asymptotics of the imaginary part of $\alpha_{n}^{j}$, we have the following:

Proposition 1.1 Assumptions and notations as in Theorem 1.2. Then

$$
\limsup _{n \rightarrow \infty}\left|\operatorname{Im} \alpha_{n}^{j}\right| \cdot \frac{2 \pi^{2}}{l_{n}^{j}}=0, \quad j=1,2, \ldots, p .
$$


We see from the above results that the limits $\liminf _{n \rightarrow \infty}\left|\operatorname{Re} \alpha_{n}^{j}\right| \cdot \frac{2 \pi^{2}}{l_{n}^{j}}, j=1,2, \ldots, p$ are the obstructions for $\left(\phi_{n}, \psi_{n}, M_{n}\right)$ to subconverge in $W^{1,2} \times L^{4}$ modulo bubbles.

Note that in Theorem 1.2, we made the assumption that all punctures of the limit surface are of Neveu-Schwarz type. However, for a general sequence of degenerating spin surfaces, it is possible that the limit spin surface has Ramond type punctures, along which the spin structure is trivial. In this case, the corresponding generalized energy identity is still open.

Now we give a brief outline of the paper. In Sect. 2, we first recall some preliminary facts about Dirac-harmonic maps from spin surfaces and then prove Theorem 1.1. In Sect. 3, some analytic properties of Dirac-harmonic maps from long spin cylinders are deduced. In Sect. 4, we study Dirac-harmonic maps from degenerating spin surfaces and show Theorem 1.2.

\section{Notations and preliminaries}

In this section, we shall first review some geometric and analytic aspects of Dirac-harmonic maps and then prove Theorem 1.1.

Let $(M, h, \mathfrak{S})$ be an oriented, compact Riemannian surface with a fixed spin structure $\mathfrak{S}$ and $P_{\text {spin(2) }} \rightarrow M$ the principal Spin(2)-bundle determined by $\mathfrak{S}$. Let $\Sigma M$ be the spinor bundle over $M$ with a hermitian metric $\langle\cdot, \cdot\rangle_{\Sigma M}$. The Levi-Civita connection $\nabla^{T M}$ on $T M$ with respect to $h$ gives rise to a connection-1-form $\left\{\omega_{\alpha \beta}\right\}_{\alpha, \beta=1}^{2}$ on $P_{\text {spin(2) }}$, and this in turn defines a spin connection $\nabla^{\Sigma M}$ on $\Sigma M$ that is compatible with $\langle\cdot, \cdot\rangle_{\Sigma M}$. For simplicity of notation, we denote $\nabla^{\Sigma M}$ by $\nabla$. The Dirac operator $\not \partial$ is locally given by $\not \partial:=e_{1} \cdot \nabla_{e_{1}} \psi+e_{2} \cdot \nabla_{e_{2}} \psi$ for a local orthonormal frame $\left\{e_{1}, e_{2}\right\}$ of $T M$ and $\psi \in \Sigma M$. We refer to [9,10,13] for more background material on spin structures and Dirac operators and to [11] for general Riemannian geometrical notations.

Let $(N, g)$ be a compact Riemannian manifold of dimension $d \geq 2$ and $\phi$ a smooth map from $M$ to $N$. By $\phi^{-1} T N$, we denote the pull-back bundle of $T N$ via $\phi$. Consider the twisted bundle $\Sigma M \otimes \phi^{-1} T N$ with a metric $\langle\cdot, \cdot\rangle_{\Sigma M \otimes \phi^{-1} T N}$ induced from the metrics on $\Sigma M$ and $\phi^{-1} T N$. There is a natural connection $\widetilde{\nabla}$ on $\Sigma M \otimes \phi^{-1} T N$ induced from those on $\Sigma M$ and $\phi^{-1} T N$, namely,

$$
\widetilde{\nabla}:=\nabla^{\Sigma M} \otimes 1+1 \otimes \nabla^{\phi^{-1} T N}
$$

The section $\psi \in \Gamma\left(\Sigma M \otimes \phi^{-1} T N\right)$ is written in local coordinates as $\psi=\psi^{i} \otimes \partial_{y^{i}}(\phi)$, where $\psi^{i} \in \Sigma M$ and $\left\{\partial_{y^{i}}\right\}$ is a local basis on $N$. The Dirac operator along the map $\phi$ is defined by

$$
\not D \psi:=e_{\alpha} \cdot \widetilde{\nabla}_{e_{\alpha}} \psi,
$$

where $\psi \in \Gamma\left(\Sigma M \otimes \phi^{-1} T N\right)$. Here and in the sequel, we apply the Einstein summation convention. Set

$$
\chi(M, N):=\left\{(\phi, \psi) \mid \phi \in C^{\infty}(M, N), \psi \in \Gamma\left(\Sigma M \otimes \phi^{-1} T N\right)\right\}
$$

and consider the following functional defined on $\chi(M, N)$ :

$$
\begin{aligned}
L(\phi, \psi) & :=\int_{M}\left(|d \phi|^{2}+\langle\psi, \not D \psi\rangle_{\Sigma M \otimes \phi^{-1} T N}\right) \operatorname{dvol}(h) \\
& =\int_{M}\left(g_{i j}(\phi) h^{\alpha \beta} \frac{\partial \phi^{i}}{\partial x_{\alpha}} \frac{\partial \phi^{j}}{\partial x_{\beta}}+g_{i j}(\phi)\left\langle\psi^{i}, \not D \psi^{j}\right\rangle_{\Sigma M}\right) \sqrt{\operatorname{det}\left(h_{\alpha \beta}\right)} d x^{1} d x^{2} .
\end{aligned}
$$


By a straightforward computation (see [6]), we get the Euler-Lagrange equations of $L$ :

$$
\begin{aligned}
\tau(\phi) & =\mathcal{R}(\phi, \psi), \\
\not D \psi & =0,
\end{aligned}
$$

where $\tau(\phi) \in \Gamma\left(\phi^{-1} T N\right)$ is the tension field of $\phi$ and $\mathcal{R}(\phi, \psi) \in \Gamma\left(\phi^{-1} T N\right)$ is defined by

$$
\mathcal{R}(\phi, \psi)(x):=\frac{1}{2} R_{l i j}^{m}(\phi(x))\left\langle\psi^{i}, \nabla \phi^{l} \cdot \psi^{j}\right\rangle \partial_{y^{m}}(\phi(x)) .
$$

Here $R_{l i j}^{m}$ are the components of the curvature tensor of $g$ and $\nabla \phi^{l} \cdot \psi^{j}$ denotes the Clifford multiplication of the vector field $\nabla \phi^{l}:=\phi_{\alpha}^{l} e_{\alpha}$ with the spinor $\psi^{j}$.

Solutions $(\phi, \psi)$ of (2.1), (2.2) are called Dirac-harmonic maps from $M$ to $N$. Thus, a Dirac-harmonic map is a map coupled with a spinor field with values in the pull-back tangent bundle. For nontrivial examples, see [6].

By the Nash-Moser embedding theorem, we embed $N$ into some $\mathbb{R}^{K}$. Let $A(\cdot, \cdot)$ be the second fundamental form of $N$ in $\mathbb{R}^{K}$ and $P(\cdot ; \cdot)$ the shape operator, satisfying $\langle P(\xi ; X), Y\rangle=$ $\langle A(X, Y), \xi\rangle$ for any $X, Y \in \Gamma(T N), \xi \in \Gamma\left(T^{\perp} N\right)$, where $T^{\perp} N$ is the normal bundle. Set

$$
\begin{aligned}
\mathcal{A}\left(d \phi\left(e_{\alpha}\right), e_{\alpha} \cdot \psi\right) & :=\phi_{\alpha}^{i} e_{\alpha} \cdot \psi^{j} \otimes A\left(\partial_{y^{i}}, \partial_{y^{j}}\right), \\
\mathcal{P}\left(\mathcal{A}\left(d \phi\left(e_{\alpha}\right), e_{\alpha} \cdot \psi\right) ; \psi\right) & :=P\left(A\left(\partial_{y^{l}}, \partial_{y^{j}}\right) ; \partial_{y^{i}}\right)\left\langle\psi^{i}, e_{\alpha} \cdot \psi^{j}\right\rangle_{\Sigma M} \phi_{\alpha}^{l} .
\end{aligned}
$$

Then Eqs. (2.1) and (2.2) become

$$
\begin{aligned}
-\Delta \phi & =A(d \phi, d \phi)+\operatorname{Re} \mathcal{P}\left(\mathcal{A}\left(d \phi\left(e_{\alpha}\right), e_{\alpha} \cdot \psi\right) ; \psi\right) \\
\not \partial & =\mathcal{A}\left(d \phi\left(e_{\alpha}\right), e_{\alpha} \cdot \psi\right) .
\end{aligned}
$$

Here, $\phi$ is a map from $M$ to $\mathbb{R}^{K}$ with

$$
\phi(x) \in N
$$

for any $x \in M$, and the spinor field $\psi$ along the map $\phi$ is a $K$-tuple of spinors $\left(\psi^{1}, \psi^{2}, \ldots, \psi^{K}\right)$ satisfying

$$
\sum_{i} v_{i} \psi^{i}=0, \text { for any normal vector } v=\sum_{i=1}^{K} v_{i} E_{i} \text { at } \phi(x),
$$

where $\left\{E_{i}, i=1,2, \ldots, K\right\}$ is the standard basis of $\mathbb{R}^{K}$.

Set

$$
\chi_{1,4 / 3}^{1,2}(M, N):=\left\{(\phi, \psi) \in W^{1,2} \times W^{1,4 / 3} \text { with (2.5) and (2.6) a.e. }\right\}
$$

Then the functional $L(\phi, \psi)$ is well-defined for $(\phi, \psi) \in \chi_{1,4 / 3}^{1,2}(M, N)$. A critical point $(\phi, \psi)$ of the functional $L$ in $\chi_{1,4 / 3}^{1,2}(M, N)$ is called a weakly Dirac-harmonic map from $M$ to $N$. When the target $N$ is the standard sphere $\mathbb{S}^{d}$, a weakly Dirac-harmonic map is smooth [5].

Let $\Omega$ be a domain of $M$. The energy of $(\phi, \psi)$ on $\Omega$ is defined by

$$
E(\phi, \psi, \Omega):=\int_{\Omega}\left(|d \phi|^{2}+|\psi|^{4}\right) .
$$


The energy of $\phi$ on $\Omega$ is

$$
E(\phi, \Omega):=\int_{\Omega}|d \phi|^{2}
$$

and the energy of $\psi$ on $\Omega$ is

$$
E(\psi, \Omega):=\int_{\Omega}|\psi|^{4} .
$$

For a two-dimensional harmonic map, there are two important geometric properties, namely, the conformal invariance and the existence of the Hopf quadratic differential. The following two propositions, proved in [6], show that these two properties are preserved in the case of Dirac-harmonic maps.

Proposition 2.1 The functional $L(\phi, \psi)$ and the energy $E(\phi, \psi)$ are conformally invariant. Namely, for any conformal diffeomorphism $f: M \rightarrow M$, set

$$
\widetilde{\phi}=\phi \circ f, \quad \widetilde{\psi}=e^{-\frac{\sigma}{2}} \psi \circ f .
$$

Then $L(\phi, \psi)=L(\widetilde{\phi}, \widetilde{\psi}), E(\phi, \psi)=E(\widetilde{\phi}, \widetilde{\psi})$. Here $e^{-\sigma}$ is the conformal factor of the conformal map $f$.

Remark 2.1 In fact, the following terms are all conformally invariant:

$$
\int|d \phi|^{2} \operatorname{dvol}(h), \quad \int\langle\psi, \not D \psi\rangle \operatorname{dvol}(h), \quad \int|\psi|^{4} \operatorname{dvol}(h) .
$$

Let $(\phi, \psi)$ be a Dirac-harmonic map from $(M, h)$. Let $\Omega \subset M$ be a small domain, and take a local isothermal coordinate $z=x+i y$ on $\Omega$ such that $h=\rho|d z|^{2}$. Define

$T(\phi, \psi)(z) d z^{2}=\left\{\left(\left|\phi_{x}\right|^{2}-\left|\phi_{y}\right|^{2}-2 i \phi_{x} \cdot \phi_{y}\right)+\left(\operatorname{Re}\left\langle\psi, \partial_{x} \cdot \psi_{x}\right\rangle-i \operatorname{Re}\left\langle\psi, \partial_{x} \cdot \psi_{y}\right\rangle\right)\right\} d z^{2}$.

Here $\partial_{x}=\frac{\partial}{\partial x}, \partial_{y}=\frac{\partial}{\partial y}, \psi_{x}=\widetilde{\nabla}_{\partial_{x}} \psi, \psi_{y}=\widetilde{\nabla}_{\partial_{y}} \psi$. Then we have

Proposition 2.2 $T(\phi, \psi)(z) d z^{2}$ is a holomorphic quadratic differential.

Now we turn to some analytic aspects of Dirac-harmonic maps. In [5] and [6], several basic properties of Dirac-harmonic maps which play an important role in the "bubbling" process were established. They can be considered as a generalization of the corresponding properties of harmonic maps. For the sake of completeness, we present them here.

Proposition 2.3 Let $(M, h)$ be a Riemann surface with a fixed spin structure and $(N, g)$ be a compact Riemannian manifold of dimension d. Then there is a small constant $\epsilon_{0}>0$ such that if $(\phi, \psi): M \rightarrow N$ is a smooth Dirac-harmonic map satisfying

$$
\int_{M}\left(|d \phi|^{2}+|\psi|^{4}\right)<\epsilon_{0},
$$

then $\phi$ is constant and consequently $\psi$ is a d-tuple of harmonic spinors. 
Theorem 2.1 ( $\epsilon$-regularity theorem) There is a small constant $\epsilon_{0}>0$ such that if $(\phi, \psi)$ : $\left(D, \delta_{\alpha \beta}\right) \rightarrow\left(N, g_{i j}\right)$ is a smooth Dirac-harmonic map satisfying

$$
\int_{D}\left(|d \phi|^{2}+|\psi|^{4}\right)<\epsilon_{0},
$$

then

$$
\begin{aligned}
\|d \phi\|_{\widetilde{D}, 1, p} & \leq C(\widetilde{D}, p)\|d \phi\|_{D, 0,2}, \\
\|\nabla \psi\|_{\widetilde{D}, 1, p} & \leq C(\widetilde{D}, p)\|\psi\|_{D, 0,4}, \\
\|\nabla \psi\|_{L^{\infty}(\widetilde{D})} & \leq C(\widetilde{D})\|\psi\|_{D, 0,4}, \\
\|\psi\|_{L^{\infty}(\widetilde{D})} & \leq C(\widetilde{D})\|\psi\|_{D, 0,4},
\end{aligned}
$$

$\forall \widetilde{D} \subset \subset D, p>1$, where $C(\widetilde{D}, p)>1$ is a constant depending only on $\widetilde{D}, p$, and the geometry of $N$.

Before we state the theorem on the removability of isolated singularities, let us recall some facts about the spin structures on surfaces (c.f. [1], Sect. 2).

Let $(M, h)$ be an oriented Riemannian surface and $P_{S O(2)}$ its oriented orthonormal frame bundle. Let $\gamma: S^{1} \rightarrow M$ be an immersion. Then the unit tangent vector field of $\gamma$ together with the corresponding unit normal vector field forms a section of $P_{S O(2)}$ along $\gamma$. A spin structure of $M$ is said to be trivial along $\gamma$ if this section lifts to a closed curve in $P_{\operatorname{spin}(2)}$; otherwise, it is said to be nontrivial along $\gamma$. It should be remarked that this notion is invariant under deformations of $\gamma$ within the same homotopy class of immersions and hence can be used to specify the two different spin structures on an annulus or a cylinder. There are various equivalent definitions of the triviality of a spin structure along a cylindrical end, see for instance [3].

Now we consider a punctured disk $D \backslash\{0\}$ with the spin structure being nontrivial along $\partial D$. Note that this spin structure extends to the unique spin structure on $D$ (c.f. [1], Sect. 2). Then we have

Theorem 2.2 (Removable singularity theorem) Let $(\phi, \psi)$ be a solution of (2.1) and (2.2) which is $C^{\infty}$ on $D \backslash\{0\}$. If $(\phi, \psi)$ has finite energy, then $(\phi, \psi)$ extends to a $C^{\infty}$ solution on D.

Remark 2.2 Here, the singularity $\{0\}$ is said to be of Neveu-Schwarz type (see [12] for an algebraic geometric description). However, there is another spin structure on $D \backslash\{0\}$ that cannot be extended to the unique spin structure on $D$, and the singularity $\{0\}$ then is said to be of Ramond type [12]. We do not know whether an analogous theorem for the Ramond type singularities also holds.

For the proofs of Proposition 2.3, Theorems 2.1 and 2.2, see [6].

Applying the geometric and analytic properties of Dirac-harmonic maps developed before, Chen et al. [5] and Zhao [20] studied the compactness of a sequence of smooth Diracharmonic maps from a fixed domain and proved the following energy identity theorem.

Theorem 2.3 Let $\left(\phi_{n}, \psi_{n}\right):(M, h, \mathfrak{S}) \rightarrow N$ be a sequence of smooth Dirac-harmonic maps with uniformly bounded energy $E\left(\phi_{n}, \psi_{n}\right) \leq \Lambda<+\infty$. Then there exist finitely many blow-up points $\left\{x_{1}, x_{2}, \ldots, x_{I}\right\}$, finitely many Dirac-harmonic maps $\left(\sigma^{i, l}, \xi^{i, l}\right): S^{2} \rightarrow$ $N, i=1,2, \ldots, I ; l=1,2, \ldots, L_{i}$, and a Dirac-harmonic map $(\phi, \psi):(M, h, \mathfrak{S}) \rightarrow N$ 
such that, after selection of a subsequence, $\left(\phi_{n}, \psi_{n}\right) \rightarrow(\phi, \psi)$ in $C_{l o c}^{\infty} \times C_{l o c}^{\infty}$ on $M \backslash\left\{x_{1}, x_{2}, \ldots, x_{I}\right\}$, and the following holds:

$$
\begin{aligned}
& \lim _{n \rightarrow \infty} E\left(\phi_{n}\right)=E(\phi)+\sum_{i=1}^{I} \sum_{l=1}^{L_{i}} E\left(\sigma^{i, l}\right), \\
& \lim _{n \rightarrow \infty} E\left(\psi_{n}\right)=E(\psi)+\sum_{i=1}^{I} \sum_{l=1}^{L_{i}} E\left(\xi^{i, l}\right) .
\end{aligned}
$$

Remark 2.3 When the domain is fixed, the "bubbling" procedure corresponds to collapsing homotopically trivial simple closed curves on the domain surface. During this process, some necks joining one bubble to the next appear, as in the case of harmonic maps. On the one hand, by applying the standard "blow-up" analysis on cylinders (c.f. Theorem 3.6 in [5]), we can obtain Dirac-harmonic maps from $\mathbb{R} \times S^{1}$. On the other hand, since any spin structure on a surface is nontrivial along any homotopically trivial simple closed curve (c.f. [1], Sect. 2), the induced spin structure on each $\mathbb{R} \times S^{1}$ is nontrivial. Also, the induced spin structures on the domain cylinders of the necks are nontrivial. Note that the nontrivial spin structure on $\mathbb{R} \times S^{1}$ can be conformally compactified to the unique spin structure on $S^{2}$. Thus, one can apply the conformal invariance of Dirac-harmonic maps and the removable singularity theorem to obtain Dirac-harmonic maps from $S^{2}$. The nontriviality of the spin structures along the domain cylinders is crucial here.

It is interesting to ask what happens when the domain of the Dirac-harmonic maps $\left(\phi_{n}, \psi_{n}\right)$ varies. By Riemann surface theory, we can fix the topological type of the surface and let the complex structure of the surface vary with $n$. The conformal invariance of Dirac-harmonic maps allows us to take a particular metric within the same conformal class. To do this, we consider closed Riemann surfaces of genus $g>1$. It follows from the uniformization theorem that any such surface acquires a complete hyperbolic metric that is unique in the conformal class determined by the complex structure. Thus, we have the following data associated to a spin surface:

$$
(M, h, c, \mathfrak{S}) .
$$

Here, $c$ is a complex structure, $h$ is the hyperbolic metric compatible with $c$ and $\mathfrak{S}$ is a spin structure.

Now, we consider a sequence of smooth Dirac-harmonic maps

$$
\left(\phi_{n}, \psi_{n}\right):\left(M_{n}, h_{n}, c_{n}, \mathfrak{S}_{n}\right) \rightarrow N
$$

with uniformly bounded energy $E\left(\phi_{n}, \psi_{n}, M_{n}\right) \leq \Lambda<\infty$.

Let us consider the simpler case that $\left(M_{n}, h_{n}, c_{n}\right)$ converges to a closed hyperbolic Riemann surface $(M, h, c)$ of the same topological type. Then there exists a sequence of diffeomorphisms $\tau_{n}: M \rightarrow M_{n}$ such that $\left(\tau_{n}^{*} h_{n}, \tau_{n}^{*} c_{n}\right)$ converges to $(h, c)$ in $C^{\infty}$ (c.f. [22]). We need to consider the change of the spin structure involved. In general, a diffeomorphism between two spin surfaces may not preserve the spin structures. However, for a closed Riemann surface of genus $g$, there are exactly $2^{2 g}$ topologically different equivalence classes of spin structures [13]. Hence, after passing to a subsequence, we can assume that the diffeomorphisms $\tau_{n}$ are compatible with the spin structures $\mathfrak{S}_{n}$, namely, the pull back of $\mathfrak{S}_{n}$ via $\tau_{n}$ is a fixed spin structure on $M$. We denote it by $\mathfrak{S}$. Recall that to fix a spin structure means to fix the equivalence class of a spin structure, thus the corresponding principle spin(2)-bundles can be naturally identified with each other via bundle isomorphisms (c.f. [4] or [15]). 
Likewise, the corresponding associated spinor bundles can also be identified with each other. As explained in [14], we think of the principle $\operatorname{spin}(2)$-bundle $P_{\operatorname{spin}(2)}$ as a topological fiber bundle and $\Sigma M$ as the associated bundle with a natural hermitian metric $\langle\cdot, \cdot\rangle_{\Sigma M}$. They are independent of the metric $h$ chosen, as long as the spin structure is fixed. The metric $h$ enters in defining the connection-1-form $\left\{\omega_{\alpha \beta}\right\}$ and hence the spin connection $\nabla^{\Sigma M}$. Thus, we can fix the spinor bundle $\Sigma M$ and think of the hyperbolic metrics $h_{n}$ and the compatible complex structures $c_{n}$ as all living on the limit surface $M$ and converging in $C^{\infty}$ to $h$ and $c$, respectively. Let $\nabla_{n}$ be the connection on $\Sigma M$ coming from $h_{n}$ and $\nabla$ be the connection on $\Sigma M$ coming from $h$. Replaced by the pullbacks, we think of $\left(\phi_{n}, \psi_{n}\right) \in C^{\infty}(M, N) \times C^{\infty}\left(\Sigma M \otimes \mathbb{R}^{K}\right)$ as a sequence of Dirac-harmonic maps defined on $\left(M, h_{n}, c_{n}, \mathfrak{S}\right)$ with respect to $\left(c_{n}, \nabla_{n}\right)$.

Proof of Theorem 1.1 As $n \rightarrow \infty,\left(h_{n}, c_{n}\right)$ converges in $C^{\infty}$ to $(h, c)$. Hence, all geometric data associated to $\left(h_{n}, c_{n}\right)$ converge in $C^{\infty}$ to those associated to $(h, c)$. In particular, the tensor $\nabla_{n}-\nabla \in \operatorname{End}\left(\Sigma M, \Sigma M \otimes T^{*} M\right)$ converges to zero in $C^{\infty}$ and the energy functional corresponding to $\left(c_{n}, \nabla_{n}\right)$ is uniformly equivalent to the one corresponding to $(c, \nabla)$. By the uniform energy bound $E\left(\phi_{n}, \psi_{n}, M_{n}\right) \leq \Lambda$, we can assume that $\left(\phi_{n}, \psi_{n}\right)$ weakly converges to some $(\phi, \psi)$ in $W^{1,2}(M, N) \times L^{4}\left(\Sigma M \otimes \mathbb{R}^{K}\right)$ with respect to $(c, \nabla)$. Note that all estimates in Proposition 2.3, Theorems 2.1 and 2.3 are uniform for the metrics $h_{n}$ and the complex structures $c_{n}$. Hence, by the standard covering argument (c.f. [17] and Theorem 2.3 in [18]), there exist finitely many points $\left\{x_{1}, x_{2}, \ldots, x_{I}\right\}$ in $M$ such that $\left(\phi_{n}, \psi_{n}\right)$ subconverges in $C_{l o c}^{\infty} \times C_{l o c}^{\infty}$ to $(\phi, \psi)$ on $M \backslash\left\{x_{1}, x_{2}, \ldots, x_{I}\right\}$. By the smoothness of $\left(\phi_{n}, \psi_{n}\right)$, we know that $(\phi, \psi) \in W^{1,2}(M, N) \times L^{4}\left(\Sigma M \otimes \mathbb{R}^{K}\right)$ is actually a smooth Dirac-harmonic map defined on $M \backslash\left\{x_{1}, x_{2}, \ldots, x_{I}\right\}$ with respect to $(c, \nabla)$. By the removable singularity theorem, $(\phi, \psi)$ extends to a smooth Dirac-harmonic map from $M$ with respect to $(c, \nabla)$. The rest of the proof of the theorem is almost immediate from applying the "blow-up" process to capture the energy concentration at the isolated singularities, which is analogous to the proof of Theorem 2.3 (see [5,20]), since all estimates are uniform for $\left(h_{n}, c_{n}\right)$.

\section{Dirac-harmonic maps from spin cylinders}

In this section, we establish a series of analytic properties of Dirac-harmonic maps from spin cylinders.

For later use, we introduce a conformal transformation between an annulus and a cylinder. Let $(r, \theta)$ be the polar coordinates of $\mathbb{R}^{2}$ centered at 0 and $h_{\text {eucl }}=d r^{2}+r^{2} d \theta^{2}$ the Euclidean metric on $\mathbb{R}^{2}$. Consider a map $f: \mathbb{R}^{1} \times S^{1} \rightarrow \mathbb{R}^{2}$ given by

$$
r=e^{-t}, \quad \theta=\theta, \quad(t, \theta) \in \mathbb{R}^{1} \times S^{1} .
$$

Let us equip $\mathbb{R}^{1} \times S^{1}$ with the metric $d s^{2}=d t^{2}+d \theta^{2}$. Then it is easy to verify that

$$
f^{*} h_{\mathrm{eucl}}=e^{-2 t} d s^{2} \text {. }
$$

Thus $f: \mathbb{R}^{1} \times S^{1} \rightarrow \mathbb{R}^{2}$ is a conformal transformation. Given $r_{1}>r_{2}$, then, the annulus $A_{r_{1}, r_{2}}:=\left\{r e^{i \theta} \mid r_{2} \leq r \leq r_{1}\right\}$ is mapped to the cylinder $P_{t_{1}, t_{2}}:=\left[t_{1}, t_{2}\right] \times S^{1}$, where $t_{i}=-\log r_{i}, i=1,2$.

Let $(\phi, \psi)$ be a Dirac-harmonic map defined on the annulus $A_{r_{1}, r_{2}} \subset \mathbb{R}^{2}$. Set

$$
\Phi:=f^{*} \phi, \quad \Psi:=e^{-\frac{t}{2}} f^{*} \psi .
$$

Then by the conformal invariance of Dirac-harmonic maps, $(\Phi, \Psi)$ is a Dirac-harmonic map defined on the cylinder $P_{t_{1}, t_{2}} \subset \mathbb{R}^{1} \times S^{1}$. 
By $P_{T_{1}, T_{2}}=\left[T_{1}, T_{2}\right] \times S^{1}$, we denote a cylinder with metric $d s^{2}=d t^{2}+d \theta^{2}$ and with the spin structure being nontrivial along the boundary curves.

The following lemma is a cylindrical version of Lemma 3.2 in [20]:

Lemma 3.1 Let $(\Phi, \Psi) \in C^{\infty}\left(P_{T_{1}, T_{2}}, N\right)$ be a Dirac-harmonic map, where $T_{2}-1>T_{1}>$ 0 . Then we have

$$
\begin{aligned}
&\left(\int_{P_{T_{1}, T_{2}-1}}|\Psi|^{4}\right)^{\frac{1}{4}} \leq C_{0}\left(\int_{P_{T_{1}, T_{2}}}|d \Phi|^{2}\right)^{\frac{1}{2}}\left(\int_{P_{T_{1}, T_{2}}}|\Psi|^{4}\right)^{\frac{1}{4}}+C\left(\int_{P_{T_{2}-1, T_{2}}}|\Psi|^{4}\right)^{\frac{1}{4}} \\
&+C\left(\int_{S_{1}}|\nabla \Psi|^{\frac{4}{3}}\right)^{\frac{3}{4}}+C\left(\int_{S_{1}}|\Psi|^{4}\right)^{\frac{1}{4}}, \\
&\left(\int_{\left.P_{T_{1}, T_{2}-1}|\nabla \Psi|^{\frac{4}{3}}\right)^{\frac{3}{4}} \leq} C_{0}\left(\int_{P_{T_{1}, T_{2}}|d \Phi|^{2}}\right)^{\frac{1}{2}}\left(\int_{P_{T_{1}, T_{2}}}|\Psi|^{4}\right)^{\frac{1}{4}}+C\left(\int_{P_{T_{2}-1, T_{2}}}|\Psi|^{4}\right)^{\frac{1}{4}}\right. \\
&+C\left(\int_{S_{1}}|\nabla \Psi|^{\frac{4}{3}}\right)^{\frac{3}{4}}+C\left(\int_{S_{1}}|\Psi|^{4}\right)^{\frac{1}{4}}
\end{aligned}
$$

where $S_{i}=\left\{T_{i}\right\} \times S^{1}, i=1,2$, and $C_{0}, C$ are constants that do not depend on $T_{1}$ and $T_{2}$.

Proof The result follows from applying the conformal transformation (3.1) to Lemma 3.2 in [20].

Moreover, let $q(t)$ be an $\mathbb{R}^{K}$-valued linear function on $\left[T_{1}, T_{2}\right]$ such that $q\left(T_{i}\right)$ equals the mean value of $\Phi$ over $S_{i}, i=1,2$. By employing the technique used by Sacks and Uhlenbeck in [17], we have the following lemma, which is part of Lemma 3.3 in [20].

Lemma 3.2 Let $(\Phi, \Psi)$ be a Dirac-harmonic map defined on $P_{T_{1}, T_{2}}$, where $T_{2}>T_{1}>0$. Then we have

$$
\begin{aligned}
\int_{P_{T_{1}, T_{2}}}\left|\Phi_{\theta}\right|^{2} \leq & C \sup _{P_{T_{1}, T_{2}}}|\Phi-q| \int_{P_{T_{1}, T_{2}}}|d \Phi|^{2}+C \sup _{P_{T_{1}, T_{2}}}|\Phi-q| \int_{P_{T_{1}, T_{2}}}|\Psi|^{4} \\
& +\int_{S_{1}}-\int_{S_{2}}(\Phi-q) \Phi_{t} d \theta .
\end{aligned}
$$

Here $C$ is a constant that only depends on $N$, not on $T_{1}$ and $T_{2}$.

For a proof, see [20]. Note that here we only estimate the vertical energy. Inspired by the proof of Theorem 3.5 in [20], we give the following lemma. 
Lemma 3.3 There exists $\epsilon_{1}>0$ such that if $(\Phi, \Psi)$ is a Dirac-harmonic map defined on $P_{T_{1}-1, T_{2}+1}$ and

$$
\begin{gathered}
\int_{P_{T_{1}-1, T_{2}+1}}|d \Phi|^{2}+|\Psi|^{4} \leq \Lambda<\infty, \\
\omega:=\sup _{t \in\left[T_{1}-1, T_{2}\right]} \int_{[t, t+1] \times S^{1}}|d \Phi|^{2}+|\Psi|^{4} \leq \epsilon_{1},
\end{gathered}
$$

then

$$
\int_{P_{T_{1}, T_{2}}}\left|\Phi_{\theta}\right|^{2}+\int_{P_{T_{1}, T_{2}}}|\Psi|^{4}+\int_{P_{T_{1}, T_{2}}}|\nabla \Psi|^{\frac{4}{3}} \leq C(\Lambda) \omega^{\frac{1}{3}} .
$$

Here, $C(\Lambda)$ is a constant depending only on $\Lambda$, but not on $T_{1}, T_{2}$.

Proof Let $\epsilon_{1}=\min \left\{\epsilon_{0}, \frac{1}{8 C_{0}^{2}}, 1\right\}$, where $\epsilon_{0}$ is the constant in the $\epsilon$-regularity theorem and $C_{0}$ is the constant in Lemma 3.1. Then the assumption (3.6) implies

$$
\sup _{t \in\left[T_{1}-1, T_{2}\right]} \int_{[t, t+1] \times S^{1}}|d \Phi|^{2}+|\Psi|^{4} \leq \epsilon_{1} \leq \frac{1}{8 C_{0}^{2}} .
$$

Since $\mu(t):=\int_{\left[T_{1}, t\right] \times S^{1}}|d \Phi|^{2}$ is a continuous and nondecreasing function on $\left[T_{1}, T_{2}\right]$ and the energy of $\Phi$ over $P_{T_{1}-1, T_{2}+1}$ is bounded by $\Lambda$, we can separate $P_{T_{1}, T_{2}}$ into finitely many parts (c.f. [20], p. 134 or similar arguments in [19], p. 689)

$$
P_{T_{1}, T_{2}}=\bigcup_{n=1}^{N_{0}} P^{n}, P^{n}:=\left[T^{n-1}, T^{n}\right] \times S^{1}, T^{0}=T_{1}, T^{N_{0}}=T_{2}
$$

such that $N_{0}$ is an integer no larger than $\left[8 C_{0}^{2} \Lambda\right]+1$, and

$$
E\left(\Phi ; P^{n}\right) \leq \frac{1}{4 C_{0}^{2}}, \quad n=1,2, \ldots, N_{0} .
$$

On each part $P^{n}$, by Lemma 3.1, we have

$$
\begin{aligned}
\left(\int_{P^{n}}|\Psi|^{4}\right)^{\frac{1}{4}} \leq & C_{0}\left(\int_{\left[T^{n-1}, T^{n}+1\right] \times S^{1}}|d \Phi|^{2}\right)^{\frac{1}{2}}\left(\int_{\left[T^{n-1}, T^{n}+1\right] \times S^{1}}|\Psi|^{4}\right)^{\frac{1}{4}} \\
& +C\left(\int_{\left[T^{n}, T^{n}+1\right] \times S^{1}}|\Psi|^{4}\right)^{\frac{1}{4}} \\
& +C\left(\int_{T^{n-1} \times S^{1}}|\nabla \Psi|^{\frac{4}{3}}\right)^{\frac{3}{4}}+C\left(\int_{T^{n-1} \times S^{1}}|\Psi|^{4}\right)^{\frac{1}{4}}
\end{aligned}
$$


Note that $\left[T^{n-1}, T^{n}+1\right] \times S^{1}=P^{n} \cup\left(\left[T^{n}, T^{n}+1\right] \times S^{1}\right)$. Hence, by the following inequalities:

$$
(a+b)^{\frac{1}{2}} \leq\left(a^{\frac{1}{2}}+b^{\frac{1}{2}}\right), \quad(a+b)^{\frac{1}{4}} \leq\left(a^{\frac{1}{4}}+b^{\frac{1}{4}}\right), \quad \forall a, b \geq 0 .
$$

and Hölder's inequality, it is easy to verify that

$$
\begin{aligned}
& \left(\int_{P^{n}}|\Psi|^{4}\right)^{\frac{1}{4}} \leq C_{0}\left(\int_{P^{n}}|d \Phi|^{2}\right)^{\frac{1}{2}}\left(\int_{P^{n}}|\Psi|^{4}\right)^{\frac{1}{4}} \\
& +C_{0}\left(\int_{P^{n}}|d \Phi|^{2}\right)^{\frac{1}{2}}\left(\int_{\left[T^{n}, T^{n}+1\right] \times S^{1}}|\Psi|^{4}\right)^{\frac{1}{4}} \\
& +C_{0}\left(\int_{\left[T^{n}, T^{n}+1\right] \times S^{1}}|d \Phi|^{2}\right)^{\frac{1}{2}}\left(\int_{P^{n}}|\Psi|^{4}\right)^{\frac{1}{4}} \\
& +C_{0}\left(\int_{\left[T^{n}, T^{n}+1\right] \times S^{1}}|d \Phi|^{2}\right)^{\frac{1}{2}}\left(\int_{\left[T^{n}, T^{n}+1\right] \times S^{1}}|\Psi|^{4}\right)^{\frac{1}{4}} \\
& +C\left(\int_{\left[T^{n}, T^{n}+1\right] \times S^{1}}|\Psi|^{4}\right)^{\frac{1}{4}} \\
& +C\left(\int_{T^{n-1} \times S^{1}}|\nabla \Psi|^{\frac{4}{3}}\right)^{\frac{3}{4}}+C\left(\int_{T^{n-1} \times S^{1}}|\Psi|^{4}\right)^{\frac{1}{4}} .
\end{aligned}
$$

From (3.5), (3.6), and (3.9), we can rewrite (3.11) as follows:

$$
\begin{aligned}
\left(\int_{P^{n}}|\Psi|^{4}\right)^{\frac{1}{4}} \leq & C\left(\int_{\left[T^{n}, T^{n}+1\right] \times S^{1}}|\Psi|^{4}\right)^{\frac{1}{4}}+C\left(\int_{\left[T^{n}, T^{n}+1\right] \times S^{1}}|d \Phi|^{2}\right)^{\frac{1}{2}} \\
& +C\left(\int_{T^{n-1} \times S^{1}}|\nabla \Psi|^{\frac{4}{3}}\right)^{\frac{3}{4}}+C\left(\int_{T^{n-1} \times S^{1}}|\Psi|^{4}\right)^{\frac{1}{4}}
\end{aligned}
$$

Here, $C$ also depends on $\Lambda$. Note that by assumption (3.6) and the definition of $\epsilon_{1}$,

$$
\omega:=\sup _{t \in\left[T_{1}-1, T_{2}\right]} \int_{[t, t+1] \times S^{1}}|d \Phi|^{2}+|\Psi|^{4} \leq \epsilon_{1} \leq 1 .
$$

Hence, by applying the $\epsilon$-regularity theorem to (3.12), we can conclude

$$
\int_{P^{n}}|\Psi|^{4} \leq C \omega^{2}+C \omega \leq C \omega .
$$


Similarly, we have

$$
\int_{P^{n}}|\nabla \Psi|^{\frac{4}{3}} \leq C \omega^{\frac{1}{3}}
$$

Summing up the estimates on $P^{n}$ gives

$$
\int_{P_{T_{1}, T_{2}}}|\Psi|^{4}=\sum_{n=1}^{N_{0}} \int_{P^{n}}|\Psi|^{4} \leq C N_{0} \omega \leq C(\Lambda) \omega
$$

and

$$
\int_{P_{T_{1}, T_{2}}}|\nabla \Psi|^{\frac{4}{3}}=\sum_{n=1}^{N_{0}} \int_{P^{n}}|\nabla \Psi|^{\frac{4}{3}} \leq C N_{0} \omega^{\frac{1}{3}} \leq C(\Lambda) \omega^{\frac{1}{3}} .
$$

In order to estimate $\int_{P_{T_{1}, T_{2}}}\left|\Phi_{\theta}\right|^{2}$, we again separate $P_{T_{1}, T_{2}}$ into smaller parts as follows:

$$
P_{T_{1}, T_{2}}=\bigcup_{n=1}^{N_{1}} \widetilde{P}^{n}, \widetilde{P}^{n}:=\left[\widetilde{T}^{n-1}, \widetilde{T}^{n}\right] \times S^{1}, \widetilde{T}^{0}=T_{1}, \widetilde{T}^{n}=\widetilde{T}^{n-1}+1, \widetilde{T}^{N_{1}}=T_{2} .
$$

Note that here, $N_{1}$ depends on $T_{1}, T_{2}$. Since $\widetilde{T}^{n}=\widetilde{T}^{n-1}+1$, by assumption (3.6) and the $\epsilon$-regularity theorem, we have $|\Phi-q| \leq C \omega^{\frac{1}{2}}$ on each part $\widetilde{P}^{n}$. Applying Lemma 3.2 on each part $\widetilde{P}^{n}$ and summing up the inequalities gives

$$
\int_{P_{T_{1}, T_{2}}}\left|\Phi_{\theta}\right|^{2} \leq C \omega^{\frac{1}{2}} \int_{P_{T_{1}, T_{2}}}|d \Phi|^{2}+C \omega^{\frac{1}{2}} \int_{P_{T_{1}, T_{2}}}|\Psi|^{4}+\int_{S_{1}}-\int_{S_{2}}(\Phi-q) \Phi_{t} d \theta .
$$

Since $q$ is equal to the mean value of $\Phi$ on $S_{i}$, by the Poincare inequality on $S_{i}$ and by Hölder's inequality, it is easy to verify that

$$
\int_{S_{i}}\left|(\Phi-q) \cdot \Phi_{t}\right| \leq C \int_{S_{i}}|d \Phi|^{2} .
$$

By the $\epsilon$-regularity theorem and the Sobolev imbedding theorem, we have

$$
\int_{S_{i}}|d \Phi|^{2} \leq C \omega .
$$

Combining (3.15), (3.16) and (3.17) gives

$$
\int_{P_{T_{1}, T_{2}}}\left|\Phi_{\theta}\right|^{2} \leq C \Lambda \omega^{\frac{1}{2}}+C \omega \leq C(\Lambda) \omega^{\frac{1}{3}} .
$$

Finally, by combining (3.18) with (3.13) and (3.14), we obtain

$$
\int_{P_{T_{1}, T_{2}}}\left|\Phi_{\theta}\right|^{2}+\int_{P_{T_{1}, T_{2}}}|\Psi|^{4}+\int_{P_{T_{1}, T_{2}}}|\nabla \Psi|^{\frac{4}{3}} \leq C(\Lambda) \omega^{\frac{1}{3}} .
$$

Thus we have finished the proof of Lemma 3.3. 
Lemma 3.4 Let $(\Phi, \Psi) \in C^{\infty}\left(P_{T_{1}, T_{2}}, N\right)$ be a Dirac-harmonic map. Then for $t \in\left[T_{1}, T_{2}\right]$,

$$
\int_{\{t\} \times S^{1}} T(\Phi, \Psi) d \theta
$$

is independent of $t \in\left[T_{1}, T_{2}\right]$, where

$$
T(\Phi, \Psi)=\left(\left|\Phi_{t}\right|^{2}-\left|\Phi_{\theta}\right|^{2}-2 i \phi_{t} \cdot \Phi_{\theta}\right)+\left(\operatorname{Re}\left\langle\Psi, \partial_{t} \cdot \Psi_{t}\right\rangle-i \operatorname{Re}\left\langle\Psi, \partial_{t} \cdot \Psi_{\theta}\right\rangle\right)
$$

and $T(\Phi, \Psi)(d t+i d \theta)^{2}$ is the holomorphic quadratic differential of $(\Phi, \Psi)$ on $P_{T_{1}, T_{2}}$.

Proof By Proposition 2.2, T $(\Phi, \Psi)$ is holomorphic on $P_{T_{1}, T_{2}}$. The rest of the proof is analogous to the case of harmonic maps (see Lemma 3.3 in [22]).

Definition 3.1 Let $(\Phi, \Psi) \in C^{\infty}\left(P_{T_{1}, T_{2}}, N\right)$ be a Dirac-harmonic map. Then we define a complex number

$$
\alpha\left(\Phi, \Psi, P_{T_{1}, T_{2}}\right):=\int_{\{t\} \times S^{1}} T(\Phi, \Psi) d \theta \in \mathbb{C}
$$

that is associated to $(\Phi, \Psi)$ along the cylinder $P_{T_{1}, T_{2}}$.

Remark 3.1 By Lemma 3.4, it is easy to verify that $\alpha\left(\Phi, \Psi, P_{T_{1}, T_{2}}\right)$ is well-defined. Moreover, $\forall t_{1}<t_{1}^{\prime}<t_{2}^{\prime}<t_{2}, \alpha\left(\Phi, \Psi, P_{t_{1}^{\prime}, t_{2}^{\prime}}\right)=\alpha\left(\Phi, \Psi, P_{t_{1}, t_{2}}\right)$.

Lemma 3.5 Let $(\Phi, \Psi) \in C^{\infty}\left(P_{T_{1}, T_{2}}, N\right)$ be a Dirac-harmonic map with $\alpha=\alpha\left(\Phi, \Psi, P_{T_{1}, T_{2}}\right)$ and

$$
\int_{P_{T_{1}-1, T_{2}+1}}|d \Phi|^{2}+|\Psi|^{4} \leq \Lambda<\infty .
$$

Then we have

(1)

$$
\begin{gathered}
\left|E\left(\Phi, P_{T_{1}, T_{2}}\right)-\right| \operatorname{Re} \alpha\left|\left(T_{2}-T_{1}\right)\right| \leq 2 \int_{P_{T_{1}, T_{2}}}\left|\Phi_{\theta}\right|^{2}+C \int_{P_{T_{1}, T_{2}}}|\Psi|^{4}+C \int_{P_{T_{1}, T_{2}}}|\nabla \Psi|^{\frac{4}{3}} \\
+C(\Lambda)\left(\int_{P_{T_{1}, T_{2}}}|\Psi|^{4}\right)^{\frac{1}{2}}
\end{gathered}
$$

(2)

$$
\begin{aligned}
|\operatorname{Im} \alpha|\left(T_{2}-T_{1}\right) \leq & C(\Lambda)\left(\int_{P_{T_{1}, T_{2}}}\left|\Phi_{\theta}\right|^{2}\right)^{\frac{1}{2}}+C \iint_{P_{T_{1}, T_{2}}}|\Psi|^{4}+C \int_{P_{T_{1}, T_{2}}}|\nabla \Psi|^{\frac{4}{3}} \\
& +C(\Lambda)\left(\int_{P_{T_{1}, T_{2}}}|\Psi|^{4}\right)^{\frac{1}{2}}
\end{aligned}
$$


Here $C, C(\Lambda)$ are constants independent of $T_{1}, T_{2}$, and $C(\Lambda)$ depends on $\Lambda$.

Proof By Definitions 3.1 and (3.19), we have

$$
\begin{aligned}
& \operatorname{Re} \alpha=\int_{0}^{2 \pi}\left|\Phi_{t}\right|^{2} d \theta-\int_{0}^{2 \pi}\left|\Phi_{\theta}\right|^{2} d \theta-\int_{0}^{2 \pi} \operatorname{Re}\left\langle\Psi, \partial_{t} \cdot \Psi_{t}\right\rangle d \theta, \\
& \operatorname{Im} \alpha=-2 \int_{0}^{2 \pi} \Phi_{t} \cdot \Phi_{\theta} d \theta-2 \int_{0}^{2 \pi} \operatorname{Re}\left\langle\Psi, \partial_{t} \cdot \Psi_{\theta}\right\rangle d \theta .
\end{aligned}
$$

Hence,

$$
\begin{aligned}
E\left(\Phi, P_{T_{1}, T_{2}}\right) & =\int_{T_{1}}^{T_{2}} \int_{0}^{2 \pi}\left|\Phi_{\theta}\right|^{2} d \theta d t+\int_{T_{1}}^{T_{2}} \int_{0}^{2 \pi}\left|\Phi_{t}\right|^{2} d \theta d t \\
& =2 \int_{P_{T_{1}, T_{2}}}\left|\Phi_{\theta}\right|^{2}+\operatorname{Re} \alpha \cdot\left(T_{2}-T_{1}\right)+\int_{P_{T_{1}, T_{2}}} \operatorname{Re}\left\langle\Psi, \partial_{t} \cdot \Psi_{t}\right\rangle, \\
& =\operatorname{Re} \alpha \cdot\left(T_{2}-T_{1}\right)+\int_{P_{T_{1}, T_{2}}}\left(2\left|\Phi_{\theta}\right|^{2}+\operatorname{Re}\left\langle\Psi, \partial_{t} \cdot \Psi_{t}\right\rangle\right) .
\end{aligned}
$$

Let $a=\operatorname{Re} \alpha \cdot\left(T_{2}-T_{1}\right), b=\int_{P_{T_{1}, T_{2}}}\left(2\left|\Phi_{\theta}\right|^{2}+\operatorname{Re}\left\langle\Psi, \partial_{t} \cdot \Psi_{t}\right\rangle\right)$. Then by the following inequality:

$$
|(a+b)-| a|| \leq|b|, \quad \forall a, b, a+b \geq 0
$$

we have

$$
\begin{aligned}
\left|E\left(\Phi, P_{T_{1}, T_{2}}\right)-\right| \operatorname{Re} \alpha\left|\cdot\left(T_{2}-T_{1}\right)\right| & \leq \int_{P_{T_{1}, T_{2}}}\left(2\left|\Phi_{\theta}\right|^{2}+\left|\operatorname{Re}\left\langle\Psi, \partial_{t} \cdot \Psi_{t}\right\rangle\right|\right) \\
& \leq 2 \int_{P_{T_{1}, T_{2}}}\left|\Phi_{\theta}\right|^{2}+\int{ }_{P_{T_{1}, T_{2}}}|\Psi| \cdot|\widetilde{\nabla} \Psi| \\
& \leq 2 \int_{P_{T_{1}, T_{2}}}\left|\Phi_{\theta}\right|^{2}+C \int_{P_{T_{1}, T_{2}}}|\Psi| \cdot(|\nabla \Psi|+|d \Phi| \cdot|\Psi|) \\
& \leq \int_{P_{T_{1}, T_{2}}\left|\Phi_{\theta}\right|^{2}+C \int_{P_{T_{1}, T_{2}}}|\Psi|^{4}+C \int_{P_{T_{1}, T_{2}}}|\nabla \Psi|^{\frac{4}{3}}} \\
& +C(\Lambda)\left(\int_{P_{T_{1}, T_{2}}}|\Psi|^{4}\right.
\end{aligned}
$$

Here, in the last step, we used the Cauchy inequality, (3.21) and the following inequality:

$$
a b \leq \frac{a^{4}+3 b^{\frac{4}{3}}}{4}, \quad \forall a, b \geq 0 .
$$


$C, C(\Lambda)$ are constants independent of $T_{1}, T_{2}$, and $C(\Lambda)$ depends on $\Lambda$.

By a similar argument, we can prove (3.23).

Now we consider a sequence of Dirac-harmonic maps from long spin cylinders under certain assumptions. The following proposition gives a "bubble domain and neck domain" decomposition for such sequences, which is analogous to the case of harmonic maps (c.f. Proposition 3.1 in [22]).

Proposition 3.1 Let $\left(\Phi_{n}, \Psi_{n}\right) \in C^{\infty}\left(P_{n}, N\right)$ be a sequence of Dirac-harmonic maps with $\alpha_{n}:=\alpha\left(\Phi_{n}, \Psi_{n}, P_{n}\right)$, where $P_{n}=\left[T_{n}^{1}, T_{n}^{2}\right] \times S^{1}$ equipped with the nontrivial spin structure. Assume that:

(1) Long cylinder property

$$
1 \ll T_{n}^{1} \ll T_{n}^{2}, \text { i.e., } \lim _{n \rightarrow \infty} \frac{1}{T_{n}^{1}}=0, \lim _{n \rightarrow \infty} \frac{T_{n}^{1}}{T_{n}^{2}}=0,
$$

(2) Uniform energy bound

$$
E\left(\Phi_{n}, \Psi_{n}, P_{n}\right) \leq \Lambda<\infty
$$

(3) Asymptotic boundary conditions

$$
\lim _{n \rightarrow \infty} \omega\left(\Phi_{n}, \Psi_{n}, P_{T_{n}^{1}, T_{n}^{1}+R}\right)=\lim _{n \rightarrow \infty} \omega\left(\Phi_{n}, \Psi_{n}, P_{T_{n}^{2}-R, T_{n}^{2}}\right)=0, \quad \forall R \geq 1,
$$

where

$$
\omega\left(\Phi, \Psi, P_{T_{1}, T_{2}}\right):=\sup _{t \in\left[T_{1}, T_{2}-1\right]} \int_{[t, t+1] \times S^{1}}|d \Phi|^{2}+|\Psi|^{4} .
$$

Then, after selection of a subsequence of $\left(\Phi_{n}, \Psi_{n}, P_{n}\right)$, either

(I)

$$
\lim _{n \rightarrow \infty} \omega\left(\Phi_{n}, \Psi_{n}, P_{n}\right)=0
$$

or

(II) $\exists K>0$ independent of $n$ and $2 K$ sequences $\left\{a_{n}^{1}\right\},\left\{b_{n}^{1}\right\},\left\{a_{n}^{2}\right\},\left\{b_{n}^{2}\right\}, \ldots,\left\{a_{n}^{K}\right\},\left\{b_{n}^{K}\right\}$ such that

$$
T_{n}^{1} \leq a_{n}^{1} \ll b_{n}^{1} \leq a_{n}^{2} \ll b_{n}^{2} \leq \cdots \leq a_{n}^{K} \ll b_{n}^{K} \leq T_{n}^{2},
$$

and

$$
\left(b_{n}^{i}-a_{n}^{i}\right) \ll T_{n}^{2}, \quad i=1,2, \ldots, K .
$$

Denote

$$
\begin{gathered}
J_{n}^{j}:=\left[a_{n}^{j}, b_{n}^{j}\right] \times S^{1}, \quad j=1,2, \ldots, K, \\
I_{n}^{0}:=\left[T_{n}^{1}, a_{n}^{1}\right] \times S^{1}, I_{n}^{K}:=\left[b_{n}^{K}, T_{n}^{2}\right] \times S^{1}, I_{n}^{i}:=\left[b_{n}^{i}, a_{n}^{i+1}\right] \times S^{1}, \\
i=1,2, \ldots, K-1 .
\end{gathered}
$$

Then

(i) $\forall i=0,1, \ldots, K, \lim _{n \rightarrow \infty} \omega\left(\Phi_{n}, \Psi_{n}, I_{n}^{i}\right)=0$. The maps $\left(\Phi_{n}, \Psi_{n}\right): I_{n}^{i} \rightarrow N$ are necks corresponding to collapsing homotopically nontrivial curves. 
(ii) $\forall j=1,2, \ldots, K$, there are finitely many Dirac-harmonic maps $\left(\omega^{j, l}, \zeta^{j, l}\right)$ : $S^{2} \rightarrow N, l=1,2, \ldots, L_{j}$, such that:

$$
\begin{aligned}
& \lim _{n \rightarrow \infty} E\left(\Phi_{n}, J_{n}^{j}\right)=\sum_{l=1}^{L_{j}} E\left(\omega^{j, l}\right), \\
& \lim _{n \rightarrow \infty} E\left(\Psi_{n}, J_{n}^{j}\right)=\sum_{l=1}^{L_{j}} E\left(\zeta^{j, l}\right) .
\end{aligned}
$$

Proof Analogous to the proof of Proposition 3.1 in [22]. One should be careful about the spin structures involved. During the "bubbling" procedure, we can obtain Dirac-harmonic maps from $\mathbb{R} \times S^{1}$ which correspond to collapsing homotopically nontrivial simple closed curves on $P_{n}$. By our assumption that the spin structures on $P_{n}$ are nontrivial, the induced spin structure on each domain $\mathbb{R} \times S^{1}$ is nontrivial and thus can be conformally compactified to the unique one on $S^{2}$. Hence, by using the conformal invariance and the removability of singularities for Dirac-harmonic maps, we can get Dirac-harmonic maps from $S^{2}$. For Dirac-harmonic maps from $\mathbb{R} \times S^{1}$ corresponding to collapsing homotopically trivial simple closed curves, see Remark 2.3. The energy identities (3.33), (3.34) follow from Theorem 2.3. Note that here, for each $j$, we do not know whether the maps $\omega^{j, l}, l=1,2, \ldots, L_{j}$ are connected or not, since the bubble tree convergence of Dirac-harmonic maps from a fixed spin surface is still open.

The next lemma gives the asymptotics of the total energy of the Dirac-harmonic necks $\left(\Phi_{n}, \Psi_{n}\right): I_{n}^{i} \rightarrow N, i=0,1, \ldots, K$ as $n \rightarrow \infty$.

Main Proposition 3.1 Assumptions and notations as in Proposition 3.1. W.l.o.g., we assume that the limit $\lim _{n \rightarrow \infty}\left|\operatorname{Re} \alpha_{n}\right| \cdot\left|P_{n}\right|$ exists in $[0,+\infty]$, where $\left|P_{n}\right|=T_{n}^{2}-T_{n}^{1}$. Then we have

$$
\begin{aligned}
& \lim _{n \rightarrow \infty} \sum_{i=0}^{K} E\left(\Phi_{n}, I_{n}^{i}\right)=\lim _{n \rightarrow \infty}\left|\operatorname{Re} \alpha_{n}\right| \cdot\left|P_{n}\right|, \\
& \lim _{n \rightarrow \infty} \sum_{i=0}^{K} E\left(\Psi_{n}, I_{n}^{i}\right)=0 .
\end{aligned}
$$

Proof We write

$$
\begin{aligned}
\sum_{i=0}^{K} E\left(\Phi_{n}, I_{n}^{i}\right) & =\sum_{i=0}^{K}\left|\operatorname{Re} \alpha_{n}\right| \cdot\left|I_{n}^{i}\right|+\sum_{i=0}^{K}\left(E\left(\Phi_{n}, I_{n}^{i}\right)-\left|\operatorname{Re} \alpha_{n}\right| \cdot\left|I_{n}^{i}\right|\right) \\
& =\mathrm{I}+\mathrm{II},
\end{aligned}
$$

where

$$
\begin{aligned}
\mathrm{I} & :=\sum_{i=0}^{K}\left|\operatorname{Re} \alpha_{n}\right| \cdot\left|I_{n}^{i}\right| \\
& =\left|\operatorname{Re} \alpha_{n}\right| \cdot\left[\left(T_{n}^{2}-T_{n}^{1}\right)-\sum_{i=1}^{K}\left(b_{n}^{i}-a_{n}^{i}\right)\right] \\
& =\left|\operatorname{Re} \alpha_{n}\right| \cdot\left(T_{n}^{2}-T_{n}^{1}\right) \cdot\left(\frac{T_{n}^{2}}{T_{n}^{2}-T_{n}^{1}}\right) \cdot\left[\left(1-\frac{T_{n}^{1}}{T_{n}^{2}}\right)-\sum_{i=1}^{K} \frac{\left(b_{n}^{i}-a_{n}^{i}\right)}{T_{n}^{2}}\right]
\end{aligned}
$$


and

$$
\mathrm{II}:=\sum_{i=0}^{K}\left(E\left(\Phi_{n}, I_{n}^{i}\right)-\left|\operatorname{Re} \alpha_{n}\right| \cdot\left|I_{n}^{i}\right|\right),
$$

By Lemmas 3.3 and 3.5 and Proposition 3.1, for $n$ large enough, we have

$$
\begin{aligned}
|\mathrm{II}| & \leq \sum_{i=0}^{K}\left|E\left(\Phi_{n}, I_{n}^{i}\right)-\right| \operatorname{Re} \alpha_{n}|\cdot| I_{n}^{i}|| \\
& \leq \sum_{i=0}^{K}\left(2 \int_{I_{n}^{i}}\left|\left(\Phi_{n}\right)_{\theta}\right|^{2}+C \int_{I_{n}^{i}}\left|\Psi_{n}\right|^{4}+C \int_{I_{n}^{i}}\left|\nabla \Psi_{n}\right|^{\frac{4}{3}}+C(\Lambda)\left(\int_{I_{n}^{i}}\left|\Psi_{n}\right|^{4}\right)^{\frac{1}{2}}\right)
\end{aligned}
$$

Note that in Proposition 3.1, after passing to subsequences, the local energy of $\left(\Phi_{n}, \Psi_{n}\right)$ over a small neighborhood of the two boundary components of $I_{n}^{i}$ can be arbitrary small. Thus, by Lemma 3.5 and Proposition 3.1,

$$
|\mathrm{II}| \leq C(\Lambda) \sum_{i=0}^{K}\left[\left(\omega\left(\Phi_{n}, \Psi_{n}, I_{n}^{i}\right)\right)^{\frac{1}{3}}+\left(\omega\left(\Phi_{n}, \Psi_{n}, I_{n}^{i}\right)\right)^{\frac{1}{6}}\right] \rightarrow 0, n \rightarrow \infty .
$$

From Proposition 3.1, we have $1 \ll T_{n}^{2} \ll T_{n}^{2}, 1 \ll\left(b_{n}^{i}-a_{n}^{i}\right) \ll T_{n}^{2}, i=1,2, \ldots, K$. Hence, by (3.38), we get $\lim _{n \rightarrow \infty} I=\lim _{n \rightarrow \infty}\left|\operatorname{Re} \alpha_{n}\right| \cdot\left|P_{n}\right|$. Now (3.35) follows immediately from (3.37) and (3.39).

Recall that $\lim _{n \rightarrow \infty} \omega\left(\Phi_{n}, \Psi_{n}, I_{n}^{i}\right)=0, i=0,1, \ldots, K$. Thus, for $n$ sufficiently large, applying Lemma 3.3 on each $I_{n}^{i}$, summing up the inequalities and finally taking the limit $(n \rightarrow \infty)$, we can prove (3.36).

By similar arguments as in the proof of Main Proposition 3.1, we get

Proposition 3.2 With the same assumptions and notations as Proposition 3.1, we have

$$
\limsup _{n \rightarrow \infty}\left|\operatorname{Re} \alpha_{n}\right| \cdot\left|P_{n}\right| \leq \Lambda, \quad \limsup _{n \rightarrow \infty}\left|\operatorname{Im} \alpha_{n}\right| \cdot\left|P_{n}\right|=0 .
$$

Proof By Lemmas 3.3 and 3.5, Proposition 3.1 and Main Proposition 3.1.

Combining the results obtained before, we state the following generalized energy identities for Dirac-harmonic maps from long spin cylinders:

Theorem 3.1 Assumptions and notations as in Main Proposition 3.1. Then there are finitely many Dirac-harmonic maps $\left(\omega^{j, l}, \zeta^{j, l}\right): S^{2} \rightarrow N, l=1,2, \ldots, L_{j} ; j=1,2, \ldots, K$, such that after selection of a subsequence of $\left(\Phi_{n}, \Psi_{n}, P_{n}\right)$, the following holds:

$$
\begin{aligned}
& \lim _{n \rightarrow \infty} E\left(\Phi_{n}, P_{n}\right)=\sum_{j=1}^{K} \sum_{l=1}^{L_{j}} E\left(\omega^{j, l}\right)+\lim _{n \rightarrow \infty}\left|\operatorname{Re} \alpha_{n}\right| \cdot\left|P_{n}\right|, \\
& \lim _{n \rightarrow \infty} E\left(\Psi_{n}, P_{n}\right)=\sum_{j=1}^{K} \sum_{l=1}^{L_{j}} E\left(\zeta^{j, l}\right) .
\end{aligned}
$$


Proof By Proposition 3.1 and Main Proposition 3.1.

As a corollary of Theorem 3.1, we have

Corollary 3.1 Assumptions and notations as in Proposition 3.1. Then $\left(\Phi_{n}, \Psi_{n}, P_{n}\right)$ subconverges in $W^{1,2} \times L^{4}$ modulo bubbles, i.e., in the limit, the necks contain no energy, if and only if

$$
\liminf _{n \rightarrow \infty}\left|\operatorname{Re} \alpha_{n}\right| \cdot\left|P_{n}\right|=0 .
$$

\section{Dirac-harmonic maps from degenerating spin surfaces}

In this section we will apply the results developed in the previous sections to prove our main theorems stated in the introduction.

To begin the proofs, we shall give a brief introduction to deformations of spin surfaces. A compact connected Riemann surface with a spin structure can be viewed as an algebraic curve with a theta characteristic, i.e., a square root of the canonical bundle $[2,16]$. The moduli space of curves with theta characteristics can be compactified algebraically by generalizing the notion of theta characteristics to the case of singular curves (a good reference is [7]).

Here, following the discussions in [3], we present a geometric topological description of the degeneration of spin surfaces. Let $\left(M_{n}, h_{n}, c_{n}, \mathfrak{S}_{n}\right)$ be a sequence of closed hyperbolic Riemann surfaces of genus $g>1$ with spin structures $\mathfrak{S}_{n}$. We assume that $\left(M_{n}, h_{n}, c_{n}\right)$ degenerates to a hyperbolic Riemann surface $(M, h, c)$ by collapsing $p(1 \leq p \leq 3 g-3)$ pairwise disjoint simple closed geodesics $\left\{\gamma_{n}^{j}, j=1,2, \ldots, p\right\}$ on $M_{n}$. For each $j$, the geodesics $\gamma_{n}^{j}$ degenerate into a pair of punctures $\left(\mathcal{E}^{j, 1}, \mathcal{E}^{j, 2}\right)$. Let $(\bar{M}, \bar{c})$ be the normalization of $(M, h, c)$. Let $\tau_{n}: M \rightarrow M_{n} \backslash \cup_{j=1}^{p} \gamma_{n}^{j}$ be the corresponding diffeomorphisms realizing the degeneration (c.f. [22]). For each $n$, the diffeomorphism $\tau_{n}$ and the spin structure $\mathfrak{S}_{n}$ together determine a pull-back spin structure on $M$. If we identify spin structures on $M$ with elements of $H^{1}(M, \mathbb{Z} / 2 \mathbb{Z})$, then a spin structure on $M$ corresponds uniquely to a spin structure on $\bar{M}$ together with a choice of an even number of punctures along which the spin structure is trivial; the induced spin structures along the remaining punctures are nontrivial (c.f. Sect. 8 in [3]). It is clear that there are finitely many spin structures on a surface with punctures. Thus, by taking subsequences, we can assume that $\tau_{n}$ is compatible with the spin structures $\mathfrak{S}_{n}$, namely the pull-back spin structure on the limit surface $M$ is fixed. Let us denote it by $\mathfrak{S}$. In particular, for each $j, \mathfrak{S}$ is nontrivial or trivial along the pair of punctures $\left(\mathcal{E}^{j, 1}, \mathcal{E}^{j, 2}\right)$ if and only if $\mathfrak{S}_{n}$ is nontrivial or trivial along the geodesic $\gamma_{n}^{j}$ for a $11 n$.

Now, we consider a sequence of smooth Dirac-harmonic maps

$$
\left(\phi_{n}, \psi_{n}\right):\left(M_{n}, h_{n}, c_{n}, \mathfrak{S}_{n}\right) \rightarrow N
$$

with uniformly bounded energy $E\left(\phi_{n}, \psi_{n}, M_{n}\right) \leq \Lambda<\infty$, where $\left(M_{n}, h_{n}, c_{n}, \mathfrak{S}_{n}\right)$ is a sequence of closed hyperbolic Riemann surfaces of genus $g>1$ with spin structures $\mathfrak{S}_{n}$. We assume that $\left(M_{n}, h_{n}, c_{n}\right)$ degenerates to a hyperbolic Riemann surface $(M, h, c)$ by collapsing $p(1 \leq p \leq 3 g-3)$ pairwise disjoint simple closed geodesics $\left\{\gamma_{n}^{j}, j=1,2, \ldots, p\right\}$ on $M_{n}$. Let $\alpha_{n}^{j}:=\alpha\left(\phi_{n}, \psi_{n}, P_{n}^{j}\right)$ be the quantities associated to $\left(\phi_{n}, \psi_{n}\right)$ along the $j$-th cylindrical collar $P_{n}^{j}$ as in Definition 3.1. By taking subsequences, we can assume that the limits

$$
\lim _{n \rightarrow \infty}\left|\operatorname{Re} \alpha_{n}^{j}\right| \cdot \frac{2 \pi^{2}}{l_{n}^{j}}, \quad j=1,2, \ldots, p
$$


exist in $[0, \infty]$. After passing to a further subsequence, we assume that the pull back of $\mathfrak{S}_{n}$ via $\tau_{n}$ is a fixed spin structure $\mathfrak{S}$ on $M$. Note that $M$ has $p$ pairs of punctures.

We require the following additional assumption:

\section{All punctures of the limit spin surface $(M, \mathfrak{S})$ are of Neveu-Schwarz type.}

It is equivalent to say that the spin structure $\mathfrak{S}$ is nontrivial along all punctures of $M$ and $\mathfrak{S}_{n}$ is nontrivial along all degenerating collars $P_{n}^{j}, j=1,2, \ldots, p$. Thus, the spin structure $\mathfrak{S}$ on $M$ extends to some spin structure $\overline{\mathfrak{S}}$ on $\bar{M}$ as explained before.

Replacing the data on $M_{n}$ by the pull-back data on $M$ and passing to subsequences, we can fix the spinor bundle $\Sigma M$ and think of the hyperbolic metrics and the compatible complex structures $\left(h_{n}, c_{n}\right)$ as all living on the limit surface $M$ and converging in $C_{l o c}^{\infty}$ to $(h, c)$. Let $\nabla_{n}$ be the connection on $\Sigma M$ coming from $h_{n}$ and $\nabla$ the connection on $\Sigma M$ coming from $h$. Then, we can consider $\left(\phi_{n}, \psi_{n}\right)$ as a sequence of Dirac-harmonic maps defined on $\left(M, h_{n}, c_{n}, \mathfrak{S}\right)$ with respect to $\left(c_{n}, \nabla_{n}\right)$.

Proof of Theorem 1.2 Analogous to the proof of Theorem 1.1 in [22]. Note that here, $\left(h_{n}, c_{n}\right)$ converges in $C_{l o c}^{\infty}$ to $(h, c)$, as $n \rightarrow \infty$. Hence, the tensor $\nabla_{n}-\nabla$ converges to zero in $C_{l o c}^{\infty}$ away from the punctures of $M$ and the energy functional corresponding to $\nabla_{n}$ over any compact subset of $M$ is uniformly equivalent to the one corresponding to $\nabla$ over the same domain. By the uniform energy bound, we can assume that $\left(\phi_{n}, \psi_{n}\right)$ subconverges weakly to a limit $(\phi, \psi)$ in $W_{l o c}^{1,2}(M, N) \times L_{l o c}^{4}\left(\Sigma M \otimes \mathbb{R}^{K}\right)$ with respect to $(c, \nabla)$. By similar arguments as in the proof of Theorem 1.1, there exist finitely many blow-up points $\left\{x_{1}, x_{2}, \ldots, x_{I}\right\}$ away from the punctures, such that $\left(\phi_{n}, \psi_{n}\right)$ subconverges to $(\phi, \psi)$ in $C_{l o c}^{\infty} \times C_{l o c}^{\infty}$ on $M \backslash\left\{x_{1}, x_{2}, \ldots, x_{I}\right\}$ with respect to $(c, \nabla)$. Furthermore, $(\phi, \psi)$ is actually a smooth Dirac-harmonic map defined on $M$ with respect to $(c, \nabla)$. Note that the complex structure $c$ on $M$ extends to some complex structure $\bar{c}$ on $\bar{M}$. By our assumption (4.1), the spin structure $\mathfrak{S}$ on $M$ extends to some spin structure $\overline{\mathfrak{S}}$ on $\bar{M}$. Hence, applying the removable singularity theorem, we have that $(\phi, \psi)$ extends to a smooth Dirac-harmonic map defined on $(\bar{M}, \bar{c}, \overline{\mathfrak{S}})$.

The energy concentration at the blow-up points $\left\{x_{1}, x_{2}, \ldots, x_{I}\right\}$ that are away from the punctures is analogous to the case in Theorem 1.1. With similar arguments as in the proof of Theorem 1.1 in [22], the energy concentration near the punctures can be reduced to the study of Dirac-harmonic maps from degenerating collars $P_{n}^{j}, j=1,2, \ldots, p$. By assumption (4.1), for all $n$, the spin structure $\mathfrak{S}_{n}$ is nontrivial along each of the degenerating collars $P_{n}^{j}, j=1,2, \ldots, p$. Thus, we can apply the results in Section 3 , especially Theorem 3.1, to capture the energy loss along the collars.

Proof of Corollary 1.1 By Theorem 1.2 and Corollary 3.1.

Proof of Proposition 1.1 By Theorem 1.2 and Proposition 3.2.

Remark 4.1 The assumption (4.1) can be satisfied by choosing suitable topological types of degeneration. For example, we consider a closed hyperbolic Riemann surface $M$ of genus $g>1$. Let $\gamma$ be a simple closed geodesic on $M$. If $M \backslash \gamma$ is disconnected, then any spin structure on $M$ must be nontrivial along the collar around $\gamma$ (c.f. Sect. 8 in [3]). In this case, pinching $\gamma$ to a point and deleting it gives a pair of Neveu-Schwarz punctures. If $M \backslash \gamma$ is connected, then the collar around $\gamma$ can carry two spin structures [3]. Hence, both types of singularities can occur when pinching $\gamma$ to a point. More generally, if $M$ carries a spin structure $\mathfrak{S}$ with Arf invariant 1 , then there is a collection of pairwise disjoint simple closed curves $\gamma_{j}: S^{1} \rightarrow M, j=1,2, \ldots, g$ representing linearly independent elements in $H_{1}(M, \mathbb{Z})$, such that $\mathfrak{S}$ is nontrivial along each of the $\gamma^{j}$ (c.f. Sect.3 in [1]). 
Acknowledgments This paper is part of the author's Ph.D. thesis [21]. He is grateful to his advisor, Prof. Jürgen Jost, for guidance and encouragement. He would also like to thank Prof. Guofang Wang, Prof. Xiaohuan Mo and Guy Buss for helpful discussions.

Open Access This article is distributed under the terms of the Creative Commons Attribution Noncommercial License which permits any noncommercial use, distribution, and reproduction in any medium, provided the original author(s) and source are credited.

\section{References}

1. Ammann, B., Bär, C.: Dirac eigenvalue estimates on surfaces. Math. Z. 240, 423-449 (2002)

2. Atiyah, M.F.: Riemann surfaces and spin structures. Ann. Sci. École Norm. Sup. 4(4), 47-62 (1971)

3. Bär, C.: The Dirac operator on hyperbolic manifolds of finite volume. J. Diff. Geom. 54, 439-488 (2000)

4. Bourguignon, J.-P., Gauduchon, P.: Spineurs, operateurs de Dirac et variations. Comm. Math. Phys. 144, 581-599 (1992)

5. Chen, Q., Jost, J., Li, J.Y., Wang, G.: Regularity and energy identities for Dirac-harmonic maps. Math. Z. 251, 61-84 (2005)

6. Chen, Q., Jost, J., Li, J.Y., Wang, G.: Dirac-harmonic maps. Math. Z. 254, 409-432 (2006)

7. Cornalba, M.: Moduli of curves and theta-characteristics. Lectures on Riemann surfaces (Trieste, 1987), pp. 560-589. World Sci. Publ., Teaneck, NJ (1989)

8. Deligne, P. et al. (eds.): Quantum Fields and Strings: A Course for Mathematicians, vols.1, 2. AMS \& Inst. Adv. Study (1999)

9. Friedrich, T.: Dirac operators in Riemannian geometry. Graduate Studies in Mathematics, vol. 25. American Mathematical Society, Providence, RI (2000), xvi+195 pp.

10. Gilkey, P.: Invariance theory, the heat equation and the Atiyah-Singer index theorem. Mathematics Lecture Series, vol. 11. Publish or Perish, Inc., Wilmington, DE, 1984, viii+349 pp

11. Jost, J.: Riemannian Geometry and Geometric Analysis, 4th edn. Universitext. Springer-Verlag, Berlin (2005). xiv+566

12. Jarvis, T.J., Kimura, T., Vaintrob, A.: Moduli spaces of higher spin curves and integrable hierarchies. Compositio Math. 126(2), 157-212 (2001)

13. Lawson, H.B., Michelsohn, M.-L.: Spin Geometry. Princeton University Press, Princeton (1989)

14. Lott, J.: $\widehat{A}$-genus and collapsing. J. Geom. Anal. 10, 529-543 (2000)

15. Maier, S.: Generic metrics and connections on spin- and spinc-manifolds. Comm. Math. Phys. 188, 407-437 (1997)

16. Mumford, D.: Theta characteristics of an algebraic curve. Ann. Sci. École Norm. Sup. 4(4), 181192 (1971)

17. Sacks, J., Uhlenbeck, K.: The existence of minimal immersions of 2-spheres. Ann. Math. 113, 1-24 (1981)

18. Sacks, J., Uhlenbeck, K.: The minimal immersions of closed Riemann surfaces. Trans. Am. Math. Soc. 271, 639-652 (1982)

19. Ye, R.G.: Gromov's compactness theorem for pseudo-holomorphic curves. Trans. Am. Math. Soc. 342(2), 671-694 (1994)

20. Zhao, L.: Energy identities for Dirac-harmonic maps. Calc. Var. Partial Differ. Equ. 28, 121-138 (2006)

21. Zhu, M.: Harmonic maps and Dirac-harmonic maps from degenerating surfaces. Ph.D. thesis, University of Leipzig (2007, submitted)

22. Zhu, M.: Harmonic maps from degenerating Riemann surfaces (2008, preprint) 